HNO 2013 · 61:494-494

DOI 10.1007/s00106-013-2708-1

Online publiziert: 29. Mai 2013

(c) Springer-Verlag Berlin Heidelberg 2013

Redaktion

M. Bloching, Berlin

\section{A.G. Beule}

Klinik und Poliklinik für HNO-Heilkunde, Kopf- und Halschirurgie, Greifswald

Telemedizinische HNO-Arzt-Konsultation

\section{Zum Beitrag}

Gollnick I, Frehiwot M, Krause EM et al (2013) Interdisziplinäre Telekonsultation in der HNOHeilkunde: Erste praktische Erfahrungen mit 100 Patienten. HNO 61:495-503 doi:s00106-012-2668-x

Die Autoren des Artikels "Interdisziplinäre Telekonsultation in der HNO-Heilkunde: Erste praktische Erfahrungen mit 100 Patienten“" stellen erste Erfahrungen mit einem Netzwerk vor, in dem Patienten aus der Praxis des Allgemeinmediziners telemedizinisch einem HNO-Arzt vorgestellt werden.

Damit wird dem breiten Kreis der HNO-Ärzte in Deutschland die Telemedizin als praxistaugliche Technologie präsentiert. Die Entwicklung hin zur virtuellen Zusammenarbeit von Ärzten ist im Kommen, sie wird nach meiner Einschätzung die HNO-Heilkunde einbeziehen. Entsprechend ist den Autoren für ihre Arbeit schon dafür zu danken, dass diese Entwicklung verstärkt in das Bewusstsein der HNO-Ärzte gelangt. Die Studie zeigt, dass technologisch eine verlässliche Übermittlung der Befunde unseres Fachgebiets heute problemlos möglich ist und sich entsprechende Netzwerke bilden können.

\section{》) Technologisch ist eine verlässliche Übermittlung der Befunde problemlos möglich}

Wie im Artikel aufgeführt, gibt es seitens der HNO-Ärzte gewisse Berührungsängste, die auch berufspolitisch motiviert sein mögen (55\% sind skeptisch, ob die Entwicklung dem Fach schadet). Demgegenüber bescheinigen die beteiligten Allgemeinärzte einen Wissenszuwachs (64 Punkte), der unseren Patienten sicherlich zugutekommen würde.

Da eine Validierung der Telemedizin noch aussteht, muss besonders auf die rechtlichen Aspekte der Aufklärung bei Anwendung von telemedizinischen Technologien und des Datenschutzes hingewiesen werden. Diese sind anderen Ortes ausführlich beleuchtet worden [1]. Auch wenn die Autoren auf die erfolgte Beratung und Aufklärung der in diese Studie eingeschlossenen Patienten leider nicht explizit hinweisen, ist diese vor Einsatz in der klinischen Praxis sehr zu empfehlen.

Zieht man die Diagnosesicherheit und bearbeiteten Fragestellungen heran (Tab. 1 des besprochenen Beitrags), so ist unklar, warum bei der Fragestellung nach einer malignen Erkrankung des Mundraums und einer Diagnosesicherheit des HNOFacharztes von 3,4 (noch „befriedigend“) für den Leser nicht eindeutig zu erkennen ist, dass sich eine konventionelle Untersuchung anschloss. Bis auf Weiteres verdient ein Patient mit der Frage nach einer malignen Entartung den Goldstandard der in Deutschland üblichen Behandlung - und die besteht in der persönlichen Untersuchung des Patienten.

Damit scheint die Telemedizin sich derzeit auf die „leichteren Fälle“ zu beschränken und hier - nach Ansicht der Autoren - zu einem vermehrten Patientenstrom zum HNO-Arzt zu führen. $\mathrm{Ob}$ das auf Dauer zutrifft und ob der Wissenszuwachs der Allgemeinärzte diesen nicht wieder verebben lässt, bleibt abzuwarten.
Zusammenfassend ist festzustellen, dass die vorliegende Arbeit das Interesse und die Auseinandersetzung von HNOÄrzten mit der Telemedizin anregen wird. Dafür ist den Autoren ausdrücklich zu danken. Alle Kollegen sind eingeladen, den zweifellos anstehenden Entwicklungsprozess zum Wohle unseres Faches $\mathrm{zu}$ begleiten und zu gestalten.

\section{Korrespondenzadresse}

PD Dr. A.G. Beule

Klinik und Poliklinik für HNO-Heilkunde, Kopf- und Halschirurgie

W.-Rathenau-Str. 43-45, 17475 Greifswald beule@uni-greifswald.de

\section{Literatur}

1. Dierks C, Feussner H, Wienke A (Hrsg) (2000) Rechtsfragen der Telemedizin. Springer, Berlin 